\title{
Enhancing Yield in Cucumber through Integrated Crop Management
}

\author{
B. Manjunath ${ }^{1 *}$, Devaraja ${ }^{2}$, Y.D. Chithra ${ }^{1}$, B. Gayathri ${ }^{2}$ and B.G. Vasanthi $^{3}$ \\ ${ }^{1}$ ICAR- Krishi Vigyan Kendra, Bengaluru Rural District, Karnataka, India \\ ${ }^{2}$ ICAR-Krishi Vigyan Kendra, Chikkaballapur, Karnataka, India \\ ${ }^{3}$ AICRP on Dryland Agriculture, UAS, GKVK, Karnataka, India \\ *Corresponding author
}

A B S T R A C T

\begin{tabular}{|l|}
\hline K e y w or d s \\
$\begin{array}{l}\text { Biocontrol agents, } \\
\text { Disease severity, } \\
\text { Fungicides, Downy } \\
\text { mildew, Management }\end{array}$ \\
\hline Article Info \\
\hline $\begin{array}{l}\text { Accepted: } \\
\text { 26 September } 2018 \\
\text { Available Online: } \\
\text { 10 October 2018 }\end{array}$ \\
\hline
\end{tabular}

\section{Introduction}

Cucumber, Cucumis sativus (L.) is one of the most preferred vegetables grown under protected conditions in the developed world. In India it is traditionally grown during January-March and September-December. Pseudoperonospora cubensis [(Berkeley \& M. A. Curtis) Rostovzev], the causal agent of cucurbit downy mildew, is responsible for devastating losses worldwide of cucumber, cantaloupe, pumpkin, watermelon and squash (Elizabeth Savory et al., 2011, Gisi and Sierotzki, 2008). According to Gisi (2002) almost $17 \%$ of the world fungicides market in 1996 was for agents used in downy mildew control. Effective control by planting resistant varieties is in many cases not possible and disease management problems have been compounded by the emergence of fungicideresistant/tolerant variants of several oomycete pathogens (Gisi, 2002; Urban and Lebeda, 2006, 2007; Urban et al., 2007).

Use of chemical fungicides leads to destruction of valuable beneficial microbes which in turn lead to increased vulnerability of crops and development of resistant strains of pathogens. Moreover, due to high input costs, crop failure by diseases results in heavy economic losses. Hence, precise disease management with emphasis on biocontrol is essential for assuring profitable farming in protected structures. 
In this context, biocontrol approaches may help develop an eco-friendly control strategy for managing plant disease (Heydari \& Misaghi 2003; Bharathi et al., 2004; Shahraki et al., 2008). Among biocontrol agents (BCA), antagonistic bacteria including Pseudomonas and Bacillus spp. have been shown to play very important roles in controlling several diseases. Therefore, the present study was undertaken to find out suitable biocontrol agents and comparatively safer fungicides to combat the disease.

Management of downy mildew in cucumber requires a multifaceted approach. At present no true resistant source of the cucumber is available in the country. Moreover, new fungicides and other molecules are introducing in the country every year against fungal diseases whose efficacy needs to be ascertained. As no true resistant cultivars are available at this moment, hence, chemical control is indispensable for alternative approach to manage these diseases. But

\section{Materials and Methods}

The field experiments for the efficacy of fungicides and biocontrol agents was conducted at, ICAR-Krishi Vigyan Kendra, Bengaluru Rural District (KVK, BRD) during Rabi 2015-16 and 2016-17 and ICAR-Krishi Vigyan Kendra, Chikkaballapur district during Rabi 2015-16 and 2016-17. The experiments were laid out in Randomized Block Design with three replications and seven treatments using cucumber hybrid Chithra. The soil of the experimental plot was red sandy loam in texture, low in available nitrogen $(241 \mathrm{~kg} / \mathrm{ha})$, medium in available phosphorus $(20.4 \mathrm{~kg} / \mathrm{ha})$ and available potassium (284 kg/ha). Normal recommended agronomic practices were adopted to grow the crop successfully in respect to application of fertilizer, irrigation and use of vegetable special. Two hand weeding was done at an interval of 25 days.
The details of treatment combinations are given in Table 1.

Trichoderma viride and Pseudomonas fluorescens were mixed with FYM and allowed to multiply with proper moisture in it for 15 days. These were applied to field just before planting. Fungicide application treatments were done by power operated sprayer. Prophylactic spray of Mancozeb $(0.25 \%)$ was given 3 weeks after sowing. Two sprays of systematic fungicides viz., Metalaxyl + Mancozeb (2.0 g /) and Dimethomorph (1.0 $\mathrm{g} / \mathrm{L})+$ Mancozeb (2.0 g /L) were applied at regular intervals fifth and seventh weeks depending on the disease severity. Fungicidal solutions were prepared by dissolving definite amount of the chemicals in definite quantity of plain water. Spray was initiated just after the detection of downy mildew symptoms in the experimental area. Care was taken during spray for both the upper and lower surface of leaves. Spray tank was thoroughly washed before filling fungicidal solution materials. Vegetable Special, crop specific micronutrient technology developed by ICAR-IIHR was spayed to plants @3g/litre of water during flowering and fruiting stage. Data were taken on foliage infection. After harvesting yield per hectare was computed.

\section{Statistical analysis}

The disease severity data was arcsine transformed before analysis of variance (ANOVA). Recorded data were subjected to statistical analysis using ANOVA of SAS statistical data analysis software. Duncan's multiple range tests was used to determine the most significant treatment.

\section{Results and Discussion}

The data on per cent disease severity of downy mildew was recorded periodically from 20 to 60 days after sowing with an interval of 10 
days (Table 2, 3, 4 and 5). Data on disease severity showed that biocontrol agents and fungicide tested reduced the disease intensity significantly compared to control. All the treatments showed different level of reaction to downy mildew of cucumber compared to control. The least per cent disease severity was recorded in $\mathrm{T}_{4}$ treatment $(7.42 \%)$ compared to check plot (24.98\%) during Rabi 2015-16 in experimental plot of ICAR - Krishi Vigyan Kendra, Bengaluru Rural District. There were significant differences within treatments on yield at study location. All treatments were exceeds the control plots. The maximum yield (34.46 tons/ha) was recorded from $\mathrm{T}_{4}$ treatment compared to check plot (28.10 t/ha). In Chikkaballapur district during Rabi 201516 the disease severity of $7.58 \%$ was recorded in $\mathrm{T}_{4}$ treatment compared to check plot $(24.98 \%)$. An yield of $34.16 \mathrm{t} / \mathrm{ha}$ was recorded form $\mathrm{T}_{4}$ treatment compared to check plot (28.65 t/ha).

Similarly during Rabi 2016- 17 in experimental plot of ICAR - Krishi Vigyan Kendra, Bengaluru Rural District the least disease severity was observed in $\mathrm{T} 4$ treatment (6.55\%) compared to check plot (22.47). Maximum yield was recorded in $\mathrm{T}_{4}$ treatment (33.76 t/ha) compared to check plot $(27.50$ t/ha). The least disease severity was recorded in $\mathrm{T}_{4}$ treatment $(7.42 \%)$ compared to check plot $(21.93 \%)$. Maximum yield was obtained in $\mathrm{T}_{4}$ treatment (34.16 t/ha) compared to check plot (27.85 t/ha) during Rabi 2016-17 in Chikkaballapur district.

Mancozeb as effective fungicide for the management of late blight and maximum fruit yield was reported by several workers (Sobolewski and Robak, 2004 and Chourasiya et al., 2013).

Results of the present study showed that all fungicide treatments significantly controlled the downy mildew infection on cucumber as compared to check plot. Duarte et al., (2007) have observed the positive effect from the combination of dimethomorph and Chlorothalonil succeeded by Metiram, compared to the combination of Metalaxyl and Chlorothalonil, succeeded by Metiram.

Table.1 Details of treatment combinations

\begin{tabular}{|l|l|}
\hline Treatments & Details \\
\hline $\mathrm{T}_{1}$-Farmer's practice & $\begin{array}{l}\text { Indiscriminate spray of fungicides viz., Mancozeb }(2.0 \mathrm{~g} / \mathrm{L}), \\
\text { Fenamidone + Mancozeb }(3.0 \mathrm{~g} / \mathrm{L}) \text {, Chlorothalonil }(2.0 \mathrm{~g} / \mathrm{L}), \\
\text { Propineb }(2.0 \mathrm{~g} / \mathrm{L}) \text {, Cymoxanil+Mancozeb }(3.0 \mathrm{~g} / \mathrm{L})\end{array}$ \\
\hline $\mathrm{T}_{2}$-Recommended practice & $\begin{array}{l}\text { Spray the crop with Metalaxyl + Mancozeb }(2.0 \mathrm{~g} / \mathrm{L}) \text { and } \\
\text { Cymoxanil+ Mancozeb }(2.0 \mathrm{~g} / \mathrm{L})\end{array}$ \\
\hline $\mathrm{T}_{3}$ - Alternate practice -1 & $\begin{array}{l}\text { Seed treatment with Captan }(2 \mathrm{~g} / \mathrm{kg} \text { seeds), Spray of Mancozeb } \\
(2.0 \mathrm{~g} / \mathrm{L}) \text { \&Cymoxanil+Mancozeb }(2.0 \mathrm{~g} / \mathrm{L})\end{array}$ \\
\hline $\mathrm{T}_{4}$ - Alternate practice -2 & $\begin{array}{l}\text { Trichoderma harzianum enriched Farm Yard Manure ( }(1 \mathrm{~kg} / \\
\text { 100 kg FYM) application } \\
\text { Seed treatment with Metalaxyl }(2 \mathrm{~g} / \mathrm{kg} \text { seeds) } \\
\text { Prophylactic Spray with Mancozeb }(2.50 \mathrm{~g} / \mathrm{L}) \text { followed by } \\
\text { Spraying of Metalaxyl+ Mancozeb }(2.0 \mathrm{~g} /) \text { and Dimethomorph } \\
\text { (1.0 g/L)+ Mancozeb (2.0 g /L) }\end{array}$ \\
\hline
\end{tabular}


Table.2 Percent disease severity and yield data of On Farm testing conducted on integrated management of downy mildew in cucumber during 2015-16 at Bengaluru Rural District

\begin{tabular}{|c|c|c|c|c|c|c|c|}
\hline \multirow{2}{*}{$\begin{array}{c}\text { Demonstration } \\
\text { number }\end{array}$} & \multicolumn{5}{|c|}{ Observations on Per cent Disease severity } & \multirow{2}{*}{$\begin{array}{l}\text { A verage } \\
\text { per cent } \\
\text { disease } \\
\text { severity }\end{array}$} & \multirow{2}{*}{$\begin{array}{l}\text { Yield } \\
\text { (t/ha) }\end{array}$} \\
\hline & 20 DAS & 30 DAS & 40 DAS & 50 DAS & 60 DAS & & \\
\hline $\mathrm{T}_{1}$ (FP) (Check) & $\begin{array}{c}16.45 \\
(23.93)\end{array}$ & $\begin{array}{c}33.95 \\
(35.63)\end{array}$ & $\begin{array}{c}30.22 \\
(33.34)\end{array}$ & $\begin{array}{c}24.68 \\
(29.77)\end{array}$ & $\begin{array}{c}19.62 \\
(26.28)\end{array}$ & $\begin{array}{c}24.98 \\
(29.79)\end{array}$ & 28.10 \\
\hline $\mathbf{T}_{2}(\mathbf{R P})$ & $\begin{array}{c}14.30 \\
(22.20)\end{array}$ & $\begin{array}{c}30.20 \\
(33.34)\end{array}$ & $\begin{array}{c}26.10 \\
(30.70)\end{array}$ & $\begin{array}{c}21.22 \\
(27.42)\end{array}$ & $\begin{array}{c}17.82 \\
(24.97)\end{array}$ & $\begin{array}{c}21.93 \\
(27.73)\end{array}$ & 29.07 \\
\hline$\overline{T_{3}(A P 1)}$ & $\begin{array}{c}16.25 \\
(23.77)\end{array}$ & $\begin{array}{c}28.02 \\
(31.95)\end{array}$ & $\begin{array}{c}22.62 \\
(28.39)\end{array}$ & $\begin{array}{c}15.29 \\
(22.98)\end{array}$ & $\begin{array}{c}11.46 \\
(19.79)\end{array}$ & $\begin{array}{c}18.73 \\
(25.38)\end{array}$ & 30.06 \\
\hline$\overline{T_{4}(\mathbf{A P 2})}$ & $\begin{array}{c}6.17 \\
(14.37)\end{array}$ & $\begin{array}{c}12.67 \\
(20.78)\end{array}$ & $\begin{array}{c}7.81 \\
(16.22)\end{array}$ & $\begin{array}{c}6.26 \\
(14.47)\end{array}$ & $\begin{array}{c}4.19 \\
(11.75)\end{array}$ & $\begin{array}{c}7.42 \\
(15.52)\end{array}$ & 34.46 \\
\hline SEm & 0.34 & 0.73 & 0.59 & 0.46 & 0.32 & 0.49 & 1.00 \\
\hline $\mathrm{CD}(0.05)$ & 1.12 & 2.39 & 1.92 & 1.51 & 1.03 & 1.59 & 3.25 \\
\hline $\mathrm{CV}$ & 7.27 & 7.45 & 7.33 & 7.36 & 7.53 & 7.39 & 7.14 \\
\hline
\end{tabular}

Table.3 Percent disease severity and yield data of On Farm testing conducted on integrated management of downy mildew in cucumber during 2016-17 at Bengaluru Rural District

\begin{tabular}{|c|c|c|c|c|c|c|c|}
\hline \multirow{2}{*}{$\begin{array}{c}\text { Demonstration } \\
\text { number }\end{array}$} & \multicolumn{5}{|c|}{ Observations on Per cent Disease severity } & \multirow{2}{*}{$\begin{array}{l}\text { Average } \\
\text { per cent } \\
\text { disease } \\
\text { severity }\end{array}$} & \multirow{2}{*}{$\begin{array}{l}\text { Yield } \\
\text { (t/ha) }\end{array}$} \\
\hline & $20 \mathrm{DAS}$ & $30 \mathrm{DAS}$ & $40 \mathrm{DAS}$ & $50 \mathrm{DAS}$ & $60 \mathrm{DAS}$ & & \\
\hline $\mathrm{T}_{1}$ (FP) (Check) & $\begin{array}{c}17.10 \\
(24.40)\end{array}$ & $\begin{array}{c}30.66 \\
(33.62)\end{array}$ & $\begin{array}{c}26.79 \\
(31.17)\end{array}$ & $\begin{array}{c}21.30 \\
(27.48)\end{array}$ & $\begin{array}{c}16.50 \\
(23.93)\end{array}$ & $\begin{array}{c}22.47 \\
(28.12)\end{array}$ & 27.50 \\
\hline $\mathbf{T}_{2}(\mathbf{R P})$ & $\begin{array}{c}17.24 \\
(24.53)\end{array}$ & $\begin{array}{c}27.93 \\
(31.90)\end{array}$ & $\begin{array}{l}24.50 \\
(29.66)\end{array}$ & $\begin{array}{c}17.60 \\
(24.80)\end{array}$ & $\begin{array}{c}13.40 \\
(21.47)\end{array}$ & $\begin{array}{c}20.13 \\
(26.47)\end{array}$ & 28.27 \\
\hline$\overline{T_{3}(A P 1)}$ & $\begin{array}{c}15.75 \\
(23.38)\end{array}$ & $\begin{array}{c}25.10 \\
(30.01)\end{array}$ & $\begin{array}{c}21.20 \\
(27.40)\end{array}$ & $\begin{array}{c}15.13 \\
(22.87)\end{array}$ & $\begin{array}{c}12.62 \\
(20.80)\end{array}$ & $\begin{array}{c}17.96 \\
(24.89)\end{array}$ & 29.25 \\
\hline $\mathrm{T}_{4}(\mathbf{A P 2})$ & $\begin{array}{c}6.72 \\
(14.98)\end{array}$ & $\begin{array}{c}9.41 \\
(17.73)\end{array}$ & $\begin{array}{c}7.28 \\
(15.65)\end{array}$ & $\begin{array}{c}5.88 \\
(14.01)\end{array}$ & $\begin{array}{c}3.44 \\
(10.68)\end{array}$ & $\begin{array}{c}6.55 \\
(14.61)\end{array}$ & 33.76 \\
\hline SEm & 0.39 & 0.74 & 0.53 & 0.40 & 0.36 & 0.48 & 1.12 \\
\hline CD (0.05) & 1.26 & 2.43 & 1.72 & 1.31 & 1.16 & 1.58 & 3.65 \\
\hline$\overline{\mathrm{CV}}$ & 6.54 & 7.99 & 6.70 & 6.98 & 8.11 & 7.26 & 8.22 \\
\hline
\end{tabular}


Table.4 Percent disease severity and yield data of on Farm testing conducted on integrated management of downy mildew in cucumber during 2015-16 at Chikkaballapur District

\begin{tabular}{|c|c|c|c|c|c|c|c|}
\hline \multirow{2}{*}{$\begin{array}{c}\text { Demonstration } \\
\text { number }\end{array}$} & \multicolumn{5}{|c|}{ Observations on Per cent Disease severity } & \multirow{2}{*}{$\begin{array}{l}\text { Average } \\
\text { per cent } \\
\text { disease } \\
\text { severity }\end{array}$} & \multirow{2}{*}{$\begin{array}{l}\text { Yield } \\
\text { (t/ha) }\end{array}$} \\
\hline & 20 DAS & 30 DAS & 40 DAS & 50 DAS & 60 DAS & & \\
\hline $\mathrm{T}_{1}(\mathrm{FP})$ (Check) & $\begin{array}{c}16.45 \\
(23.93)\end{array}$ & $\begin{array}{c}33.95 \\
(35.63)\end{array}$ & $\begin{array}{c}30.22 \\
(33.34)\end{array}$ & $\begin{array}{c}24.68 \\
(29.77)\end{array}$ & $\begin{array}{c}19.62 \\
(26.28)\end{array}$ & $\begin{array}{c}24.98 \\
(29.79)\end{array}$ & 28.65 \\
\hline $\mathbf{T}_{2}(\mathbf{R P})$ & $\begin{array}{c}15.17 \\
(22.92)\end{array}$ & $\begin{array}{c}28.31 \\
(32.14)\end{array}$ & $\begin{array}{l}24.50 \\
(29.65)\end{array}$ & $\begin{array}{c}19.60 \\
(26.27)\end{array}$ & $\begin{array}{c}14.12 \\
(22.06)\end{array}$ & $\begin{array}{c}20.34 \\
(26.61)\end{array}$ & 29.97 \\
\hline $\mathbf{T}_{\mathbf{3}}(\mathbf{A P 1})$ & $\begin{array}{c}10.95 \\
(19.30)\end{array}$ & $\begin{array}{c}25.10 \\
(30.01)\end{array}$ & $\begin{array}{c}20.07 \\
(26.61)\end{array}$ & $\begin{array}{c}16.62 \\
(24.10)\end{array}$ & $\begin{array}{c}10.15 \\
(18.57)\end{array}$ & $\begin{array}{c}16.58 \\
(23.72)\end{array}$ & 30.64 \\
\hline $\mathrm{T}_{4}(\mathrm{AP2})$ & $\begin{array}{c}5.47 \\
(13.52)\end{array}$ & $\begin{array}{c}13.47 \\
(21.50)\end{array}$ & $\begin{array}{c}9.20 \\
(17.66)\end{array}$ & $\begin{array}{c}5.86 \\
(14.00)\end{array}$ & $\begin{array}{c}3.91 \\
(11.40)\end{array}$ & $\begin{array}{c}7.58 \\
(15.62)\end{array}$ & 35.06 \\
\hline SEm & 0.34 & 0.73 & 0.59 & 0.46 & 0.32 & 0.49 & 0.95 \\
\hline CD (0.05) & 1.12 & 2.39 & 1.92 & 1.51 & 1.03 & 1.59 & 3.09 \\
\hline$\overline{C V}$ & 7.27 & 7.45 & 7.33 & 7.36 & 7.53 & 7.39 & 6.86 \\
\hline
\end{tabular}

Table.5 Percent disease severity and yield data of On Farm testing conducted on integrated management of downy mildew in cucumber during 2016-17 at Chikkaballapur District

\begin{tabular}{|c|c|c|c|c|c|c|c|}
\hline \multirow{2}{*}{$\begin{array}{c}\text { Demonstration } \\
\text { number }\end{array}$} & \multicolumn{5}{|c|}{ Observations on Per cent Disease severity } & \multirow{2}{*}{$\begin{array}{l}\text { A verage } \\
\text { per cent } \\
\text { disease } \\
\text { severity }\end{array}$} & \multirow{2}{*}{$\begin{array}{l}\text { Yield } \\
\text { (t/ha) }\end{array}$} \\
\hline & 20 DAS & 30 DAS & 40 DAS & 50 DAS & 60 DAS & & \\
\hline $\mathrm{T}_{1}$ (FP) (Check) & $\begin{array}{c}14.30 \\
(22.20)\end{array}$ & $\begin{array}{c}30.20 \\
(33.34)\end{array}$ & $\begin{array}{c}26.10 \\
(30.70)\end{array}$ & $\begin{array}{c}21.22 \\
(27.42)\end{array}$ & $\begin{array}{c}17.82 \\
(24.97)\end{array}$ & $\begin{array}{c}21.93 \\
(27.73)\end{array}$ & 27.85 \\
\hline $\mathbf{T}_{2}(\mathbf{R P})$ & $\begin{array}{c}16.25 \\
(23.77)\end{array}$ & $\begin{array}{c}28.02 \\
(31.95)\end{array}$ & $\begin{array}{c}22.62 \\
(28.39)\end{array}$ & $\begin{array}{c}15.29 \\
(22.98)\end{array}$ & $\begin{array}{c}11.46 \\
(19.79)\end{array}$ & $\begin{array}{c}18.73 \\
(25.38)\end{array}$ & 28.47 \\
\hline $\mathbf{T}_{3}$ (AP1) & $\begin{array}{c}10.95 \\
(19.30)\end{array}$ & $\begin{array}{c}25.10 \\
(30.01)\end{array}$ & $\begin{array}{c}20.07 \\
(26.61)\end{array}$ & $\begin{array}{c}16.62 \\
(24.10)\end{array}$ & $\begin{array}{c}10.15 \\
(18.57)\end{array}$ & $\begin{array}{c}16.58 \\
(23.72)\end{array}$ & 29.64 \\
\hline $\mathrm{T}_{4}(\mathbf{A P 2})$ & $\begin{array}{c}6.17 \\
(14.37)\end{array}$ & $\begin{array}{c}12.67 \\
(20.78)\end{array}$ & $\begin{array}{c}7.81 \\
(16.22)\end{array}$ & $\begin{array}{c}6.26 \\
(14.47)\end{array}$ & $\begin{array}{c}4.19 \\
(11.75)\end{array}$ & $\begin{array}{c}7.42 \\
(15.52)\end{array}$ & 34.16 \\
\hline SEm & 0.34 & 0.73 & 0.59 & 0.46 & 0.32 & 0.49 & 0.95 \\
\hline CD (0.05) & 1.12 & 2.39 & 1.92 & 1.51 & 1.03 & 1.59 & 3.33 \\
\hline CV & 7.27 & 7.45 & 7.33 & 7.36 & 7.53 & 7.39 & 7.37 \\
\hline
\end{tabular}


Table.6 Pooled data of percent disease severity and yield data of OFT conducted on integrated management of downy mildew in cucumber at Bengaluru Rural District and Chikkaballapur district

\begin{tabular}{|c|c|c|c|c|c|c|c|c|c|c|c|}
\hline \multirow{4}{*}{$\begin{array}{l}\text { Treatment } \\
\text { details }\end{array}$} & \multirow[t]{4}{*}{ Treatments } & \multicolumn{8}{|c|}{ Average Per cent Disease Index } & \multirow{4}{*}{$\begin{array}{c}\text { Poole } \\
\text { d } \\
\text { PDI }\end{array}$} & \multirow{4}{*}{$\begin{array}{l}\text { Pooled } \\
\text { yield }\end{array}$} \\
\hline & & \multicolumn{2}{|c|}{ 2014-15 } & \multirow{2}{*}{\multicolumn{2}{|c|}{ u Rural }} & \multicolumn{2}{|c|}{ 2014-15 } & \multicolumn{2}{|c|}{ 2015-16 } & & \\
\hline & & \multicolumn{2}{|c|}{ Bengaluru Rural } & & & \multicolumn{4}{|c|}{ Chikkaballapur } & & \\
\hline & & PDI & $\begin{array}{l}\text { Yield } \\
\text { (t/ha) }\end{array}$ & PDI & $\begin{array}{l}\text { Yield } \\
\text { (t/ha) }\end{array}$ & PDI & $\begin{array}{l}\text { Yield } \\
\text { (t/ha) }\end{array}$ & PDI & $\begin{array}{l}\text { Yield } \\
\text { (t/ha) }\end{array}$ & & \\
\hline $\mathbf{T}_{1}$ & $\begin{array}{l}\text { Farmers practice } \\
\text { (Check) }\end{array}$ & $\begin{array}{c}24.98 \\
(29.79)\end{array}$ & 28.10 & $\begin{array}{c}22.47 \\
(28.12)\end{array}$ & 27.50 & $\begin{array}{c}24.98 \\
(29.79)\end{array}$ & 28.65 & $\begin{array}{c}21.93 \\
(27.73)\end{array}$ & 27.85 & 23.59 & 28.03 \\
\hline $\mathbf{T}_{2}$ & $\begin{array}{l}\text { Recommended } \\
\text { practice }\end{array}$ & $\begin{array}{c}21.93 \\
(27.73)\end{array}$ & 29.07 & $\begin{array}{c}20.13 \\
(26.47)\end{array}$ & 28.27 & $\begin{array}{c}20.34 \\
(26.61)\end{array}$ & 29.97 & $\begin{array}{c}18.73 \\
(25.38)\end{array}$ & 28.47 & 20.28 & 28.95 \\
\hline $\mathbf{T}_{3}$ & $\begin{array}{l}\text { Alternate } \\
\text { practice }\end{array}$ & $\begin{array}{c}18.73 \\
(25.38)\end{array}$ & 30.06 & $\begin{array}{c}17.96 \\
(24.89)\end{array}$ & 29.25 & $\begin{array}{l}16.58 \\
(23.72)\end{array}$ & 30.64 & $\begin{array}{l}16.58 \\
(23.72)\end{array}$ & 29.64 & 17.46 & 29.90 \\
\hline $\mathbf{T}_{4}$ & $\begin{array}{l}\text { Alternate } \\
\text { practice } 2\end{array}$ & $\begin{array}{c}7.42 \\
(15.52)\end{array}$ & 34.46 & $\begin{array}{c}6.55 \\
(14.61)\end{array}$ & 33.76 & $\begin{array}{c}7.58 \\
(15.62)\end{array}$ & 35.06 & $\begin{array}{c}7.42 \\
(15.52)\end{array}$ & 34.16 & 7.24 & 34.36 \\
\hline SEm & & $\begin{array}{c}7.42 \\
(15.52)\end{array}$ & 34.46 & $\begin{array}{c}6.55 \\
(14.61)\end{array}$ & 33.76 & $\begin{array}{c}7.58 \\
(15.62)\end{array}$ & 35.06 & $\begin{array}{c}7.42 \\
(15.52)\end{array}$ & 34.16 & 7.24 & 34.36 \\
\hline CD at $5 \%$ & & 0.49 & 1.00 & 0.48 & 1.12 & 0.49 & 0.95 & 0.49 & 0.95 & 0.49 & 1.01 \\
\hline $\mathbf{C V}$ & & 1.59 & 3.25 & 1.58 & 3.65 & 1.59 & 3.09 & 1.59 & 3.33 & 1.59 & 3.33 \\
\hline
\end{tabular}

Table.7 Economics of treatments evaluated for the management of downy mildew in cucumber

\begin{tabular}{|l|l|l|l|l|l|l|}
\hline Treatments & $\begin{array}{l}\text { Yield } \\
\text { (kg/ha) }\end{array}$ & $\begin{array}{l}\text { Gross } \\
\text { returns } \\
\text { (Rs.8/kg) }\end{array}$ & $\begin{array}{l}\text { Cost of } \\
\text { cultivatio } \\
\text { n/ha }\end{array}$ & $\begin{array}{l}\text { Marginal } \\
\text { cost (Rs.) }\end{array}$ & $\begin{array}{l}\text { Total cost } \\
\text { (Rs.) }\end{array}$ & $\begin{array}{l}\text { B:C ratio } \\
\text { (GR/ } \\
\text { TC) }\end{array}$ \\
\hline $\mathrm{T}_{1}$ (Check) & 28030 & 224240 & 85000 & 6950 & 91950 & 2.44 \\
\hline $\mathrm{T}_{2}$ (Recommended) & 28950 & 231600 & 85824 & 3960 & 89784 & 2.58 \\
\hline $\mathrm{T}_{3}$ (Recommended) & 29900 & 239200 & 87500 & 4350 & 91850 & 2.73 \\
\hline $\mathrm{T}_{4}$ (Evaluated) & 34360 & 274880 & 85000 & 7100 & 92100 & 2.98 \\
\hline
\end{tabular}

The efficacy of the tested bioagents against downy mildew may be due to nutrient competition, hyperparasitism, and/or antibiosis.

The pooled data (Table 6) indicated that, the average per cent disease severity was least in $\mathrm{T}_{4}$ treatment $(7.49 \%)$ compared to Check plot (23.59\%). Maximum yield was recorded in $\mathrm{T}_{4}$ treatment (34.36 t/ha) compared to check plot (28.03 t/ha).

From the pooled data of three season, it is evident that benefit: cost ratio of 2.98 was recorded in $\mathrm{T}_{4}$ treatment and the least was noticed in check plot (2.44). A net income of Rs. 274880 /ha by investing Rs. 92100/ha was realized in $\mathrm{T}_{4}$ treatment compared to check plot (Table 7). With respect to the economics of treatments evaluated for the management of late blight in potato the yield was highest in $\mathrm{T}_{4}$ treatment (34.36 t/ha) compared to check plot (28.03 t/ha) (Table 7).

\section{Acknowledgement}

Authors are thankful to Agricultural Technology Application Research Institute (ATARI), Indian Council of Agricultural Research, Zone XI India for their kind 
guidance, motivation and financial support for this work.

\section{References}

Bharathi, R., Vivekananthan, R., Harish, S., Ramanathan, A. and Samiyappan, R. (2004). Rhizobacteria-based bioformulations for the management of fruit rot infection in chilies. Crop Protection, 23: 835-843.

Chourasiya, P. K., Lal, A.A. and Simon, S. (2013). Effect of certain fungicides and botanicals against early blight of tomato caused by Alternariasolani (Ellis and Martin) under Allahabad Uttar Pradesh, India conditions. Int. J. Agric. Sci., \& Res., 3 (3):151-156.

Duarte, H., da S. S., Zambolium, L. and Jesus Junior, W. C. (2007). Manejo darequeima do tomateiro industrial empregandosistema de previsro. Summa Phytopathologica, 33 (4): 328-334.

Elizabeth A. Savory, Leah L. Granke, Lina M. Quesada O Campo, Marina Varbanova, Mary K. Hausbeck and Brad Day. (2011). Molecular Plant Pathology, 12(3): 217-226.

Gisi, U. (2002). Chemical control of downy mildews. In P. T. N. Spencer-Phillips, U. Gisi, \& A. Lebeda (Eds.), Advances in downy mildew research (pp, Vol. 1, pp. 119-159). Dordrecht: Kluwer Academic Publishers.

Gisi, U., and Sierotzki, H. (2008). Fungicide modes of action and resistance in downy mildews. European Journal of Plant Pathology, 122: 157-167.

Heydari, A. and Misaghi, I.J. (2003): The role of rhizosphere bacteria in herbicidemediated increase in Rhizoctonia solani-induced cotton seedling damping-off. Plant and Soil, 257: 391396.

Shahraki, M., Heydari, A., Hassanzadeh, N., Rezaei, S. and Naraghi, L. (2008). Investigation on the possibility of biological control of sugar beet seedling damping-off disease. Iranian J. Agric. Sci., 13: 23-38.

Sobolewski, J. and Robak, J. (2004). New products used for complex disease control on tomato growing in open field. Progressive Plant Protec., 44: 1105-1107.

Urban, J. and Lebeda, A. (2006). Fungicide resistance in cucurbit downy mildew Methodological, biological and population aspects. Annals of Applied Biology, 149(1):63 - 75.

Urban, J. and Lebeda, A. (2007). Variation for fungicide resistance in Czech populations of Pseudoperonospora cubensis. Journal of Phytopathology, 155: 143-51.

\section{How to cite this article:}

Manjunath, B., Devaraja, Y.D. Chithra, B. Gayathri and Vasanthi, B.G. 2018. Enhancing Yield in Cucumber through Integrated Crop Management. Int.J.Curr.Microbiol.App.Sci. 7(10): 35823588. doi: https://doi.org/10.20546/ijcmas.2018.710.415 